\title{
Résultats hémodynamiques et cliniques de la dissec- tion, assistée par lunettes chirurgicales, des veines spermatiques au niveau de l'orifice inguinal interne
}

\author{
Giandomenico PASSAVANTI, Valerio PIZZUTI, Fabio Massimo COSTANTINI, \\ Riccardo PAOLINI
}

Service d'Urologie Andrologie, Hôpital “Misericordia”, Grosseto, Italie

\section{RESUME}

Le reflux veineux réno-spermatique est très bien démontré par l'échographie couleur doppler (ECCD) et il est l'élément hémodynamique de base de la varicocèle. Nous avons essayé de vérifier l'utilité de la dissection, avec des lunettes chirurgicales permettant un grossissement de 4,5 des branches de la veine spermatique au niveau de l'orifice inguinal interne.

16 patients ont été opérés selon la classification échographique de Hirsh et al. [8]. La varicocèle était du llle degré pour 2 cas, du IVe degré pour 11 cas et du Ve degré pour 3 cas. La varicocèle était à gauche chez 13 patients et à droite dans 3 cas.

Le diagnostic a été réalisé par examen clinique et ECCD. Le temps opératoire était de 25-35 min. L'incision avait 1,5 à $2 \mathrm{~cm}$ de long. Nous n'avons jamais eu de complications à court terme, ni d'hydrocèle à long terme. L'ECCD après six mois n'a pas trouvé de reflux réno-spermatique. Le volume testiculaire moyen qui était de $22 \mathrm{ml}$ avant, ne changeait pas après l'intervention chirurgicale.

Cette intervention chirurgicale est moins invasive que l'intervention rétro-péritonéale classique ou laparoscopique, et l'abord chirurgical est moins important respectivement à la technique percutanée ; elle est moins radicale que l'intervention en microchirurgie classique, mais le temps opératoire est plus bref, et la plaie chirurgicale est plus petite ; elle respecte l'artère et les vaisseaux lymphatiques.

En conclusion, cette intervention peut être considérée comme une bonne option dans le traitement chirurgical de la varicocèle parce qu'elle a peu de complications, une absence d'hydrocèle, une importance chirurgicale limitée et permet des bons résultats hémodynamiques.

Mots clés : varicocèle, varicocèlectomie, power-doppler, microchirurgie

\section{INTRODUCTION}

L'inversion du flux veineux est la caractéristique essentielle de la varicocèle [4] et son diagnostic repose sur l'examen clinique, sur le doppler et l'échographic coulcur doppler (ECCD) $[12,17]$. La chirurgie a pour but de modifier cette inversion et de sectionner les veines spermatiques et toutes ses branches collatérales [6]. Nous avons essayé de vérifier les avantages techniques et hémodynamiques de la dissection de la veine spermatique, avec l'utilisation de lunettes chirurgicales permettant un grossissement de 4,5, au niveau de l'orifice inguinal interne.

\section{Correspondance}

Dr Giandomenico Passavanti - Rue Oberdan 44 - 58100 Grosseto. Italie - Tel 0039-0564 411864 - Email mpeppina@infinito.it 


\section{MATERIEL ET METHODE}

Seize patients d'âge compris entre 14 et 41 ans (moyenne $=23,4)$, ont été opérés par dissection et section de la veine spermatique. L'indication chirurgicale a été poséc en raison d'une infécondité ( $n=8$ ), d'altérations du spermogramme de type oligo-asthénospermie $(n=5)$ ou pour des raisons esthétiques $(n=3)$. Treize patients avaient une oligo- asthénospermic et trois avaient un spermogramme normal. Selon la classification échographique de Hirsh [8], la varicocèle était du IIle degré chez deux patients, du IVe degré chez onze patients, et du Ve degré chez trois patients. La varicocèle était à gauche dans 13 cas et bilatérale pour 3. Le diagnostic reposait sur l'examen clinique et l'ECCD des vaisseaux du cordon spermatique. Le volume testiculaire a été mesuré, avant et 6 mois après la chirurgie, avec une sonde échographique $7 \mathrm{Mh}$; les trois diamètres $(\mathrm{a}, \mathrm{b}, \mathrm{c})$ ont été évalués et le volume calculé selon la formule classique $(0,52 \times$ a $\times$ b $\times c)$.

L'intervention débutait par une petite incision de $1,5-2 \mathrm{~cm}$ en regard de l'orifice inguinal interne, permettant l'exposition du cordon spermatique et le décollement du déférent, puis la dissection des branches de la veine spermatique (Figure 1), avec des lunettes chirurgicales (grossissement de 4,5 fois) pour identifier et respecter l'artère et les vaisseaux lymphatiques; enfin suture et section des vaisseaux veineux (Figure 2).

Les données ont été analysées en utilisant le test non paramétrique de Wilcoxon.

\section{RESULTATS}

Les temps opératoires étaient de 25 à 35 minutes. Nous n'avons jamais eu de complications précoces, ni d'épididymites ou de phlébites. Tous les patients ont eu un jour d'hospitalisation. Le contrôle à six mois après l'intervention n'a pas montré d'hydrocèle ; l'ECCD n'a jamais révélé de récidives de la varicocèle, et a toujours permis d'identifier l'artère spermatique. La comparaison des volumes testiculaires mesurés avant et six mois après la chirurgie montre une absence de modification du côté droit (Figure $3 \mathrm{~A})$ et une augmentation significative $(\mathrm{p}=0,01)$ mais faible du côté gauche (Figure 3B).

En ce qui concerne la spermatogénèse, la comparaison des moyennes de numérations des spermatozoïdes $/ \mathrm{ml}$ obtenues avant et six mois après chirurgie, indique une amélioration significative ( $p=0,041$ ), mais qu'il faut relativiser en prenant en compte les valcurs individuelles comme rapportées à la Figurc 4.

\section{DISCUSSION}

Le trouble hémodynamique de la varicocèle participe à la survenue de modifications thermiques [10], hormonales
[2], et métaboliques [11] responsables des anomalies du spermogramme [20]. La chirurgie est la base thérapeutique, et ses résultats peuvent être évalués par l'ECCD [5, 18]. Plusieurs techniques chirurgicales ont été proposées pour traiter la varicocèlc et toutes ont des avantages et des désavantages.

La technique classique, décrite par Ivanissevich, est le point de départ pour traiter cette chirurgie. Cette intervention, avec toutes ses variantes, prévoit un abord rétro-péritonéal de la veine spermatique [19]. Elle est compliquée par une persistance de la varicocèle dans environ $9 \%$ des cas $[4,6]$, et la fréquentc apparition d'un hydrocèle secondaire à la section des vaisseaux lymphatiques [6]. La chirurgie laparoscopique donne de bons résultats sur le plan esthétique, mais elle est associé d'une part à une incidence d'environ $17 \%$ de récidives de la varicocèle, d'autre part à la survenue d'une hydrocèle dans 14 à $24 \%$ des cas [13, 16]. Les susdites interventions peuvent souvent causer la section accidentelle de l'artère spermatique, et cet événement en général n’endommage pas le testicule, mais ne détermine quand même pas des avantages au regard de la qualité du sperme [15].

Les techniques percutanćes utilisent des abords endovasculaires importants, mais elles donnent d'excellents résultats esthétiques ; elles entraînent une récidive de la varicocèle dans $11 \%$ des cas et elles peuvent causer des phlébites du scrotum $[14]$.

Les interventions en microchirurgie peuvent avoir un abord sous-inguinal avec une exposition du testicule et du cordon, et une dissection très extensive de veines spermatiques [7], mais elles peuvent aussi avoir un abord susinguinal et une dissection de la veine spermatique, laquelle est anastomosée à la veine épigastrique [1] ou à la veine saphène [9]. Ces interventions donnent des résultats excellents $[7]$ en termes de récidives (1-2\%) et d'hydrocèle secondaire $(1 \%)$; elles ont des temps opératoires très longs et le résultat esthétique est décevant.

L'intervention rapportéc dans le présent article, exécutée avec des lunettes chirurgicales, comprend une dissection vasculaire très limitée, mais elle ne prévoit pas une anastomose vasculaire ; elle a des temps opératoires très brefs (25-35 minutes), une plaie chirurgicale de petite taille, l'hydrocèle postopératoire en est presque absente. et l'incidence des récidives est très faible [3].

\section{CONCLUSION}

Selon notre expérience, empirique mais à notre avis intéressante, on peut estimer que la dissection décrite présente l'avantage d'être peu invasive et de donner des bons résultats sur le plan hémodynamique, sur le plan esthétique, sur le plan des durées opératoires et d'hospitalisation ; elle peut donc être proposée pour traiter la varicocèle, surtout des IIIe et IVe degrés. 


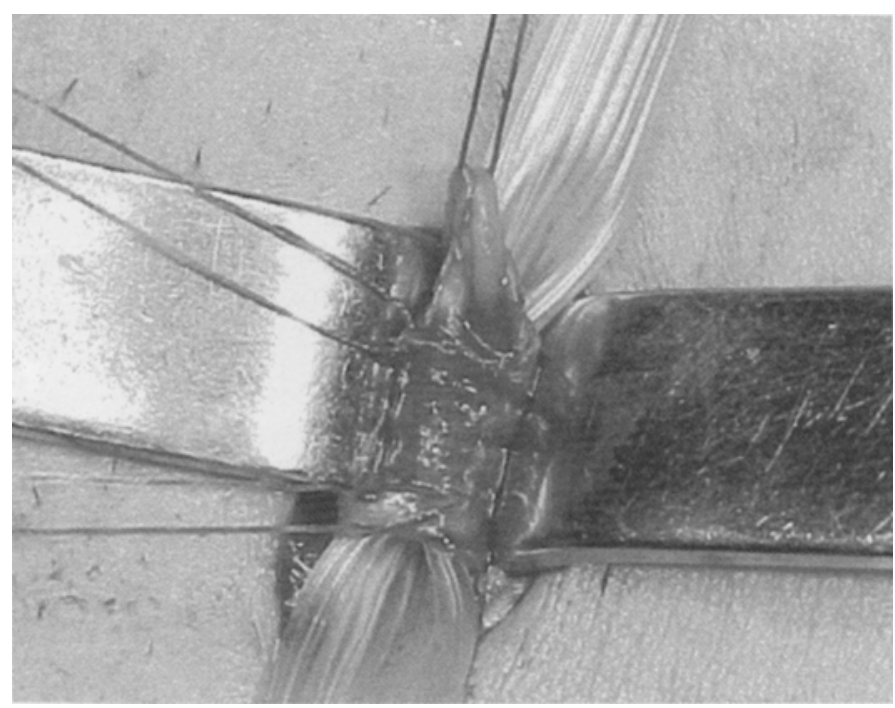

Figure 1: Dissection des branches de la veine spermatique avec lunette chirurgicale pour identifier et respecter l'artère et les vaisseaux lymphatiques.

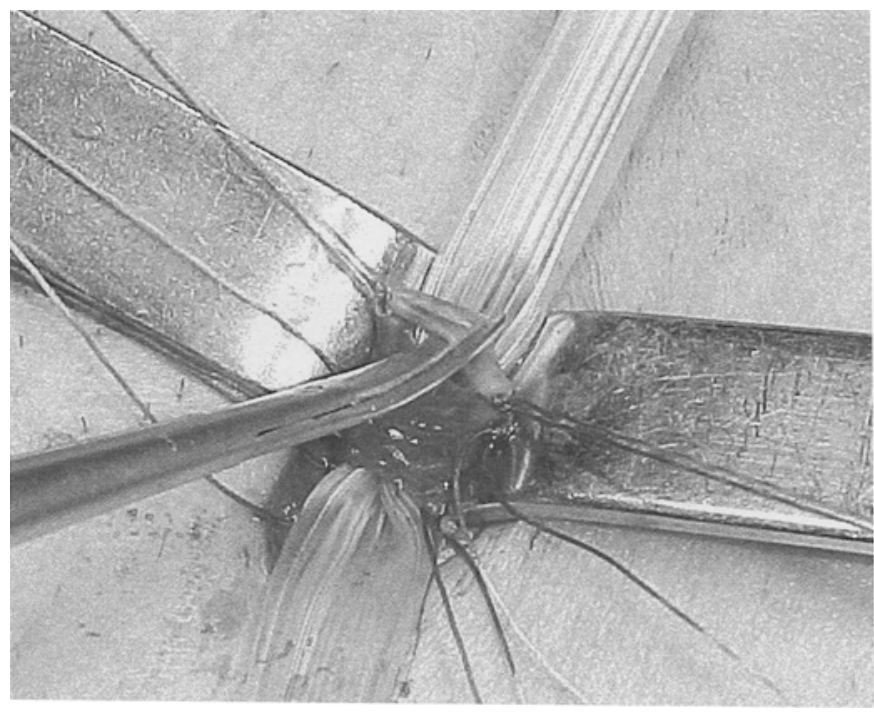

Figure 2 : Section et ligature des vaisseaux veineux.

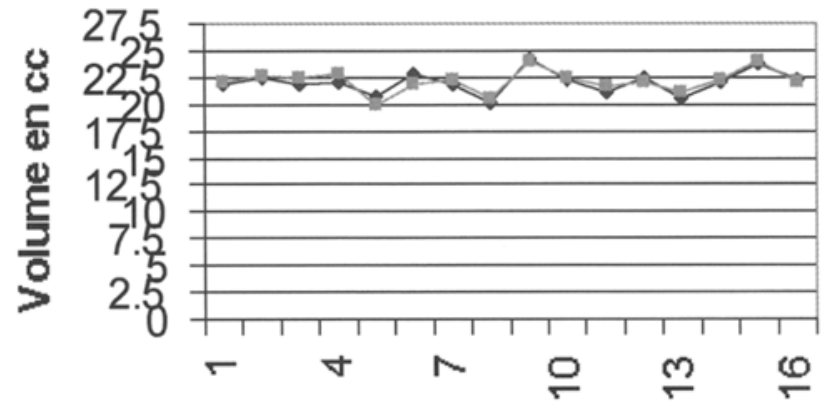

Pts

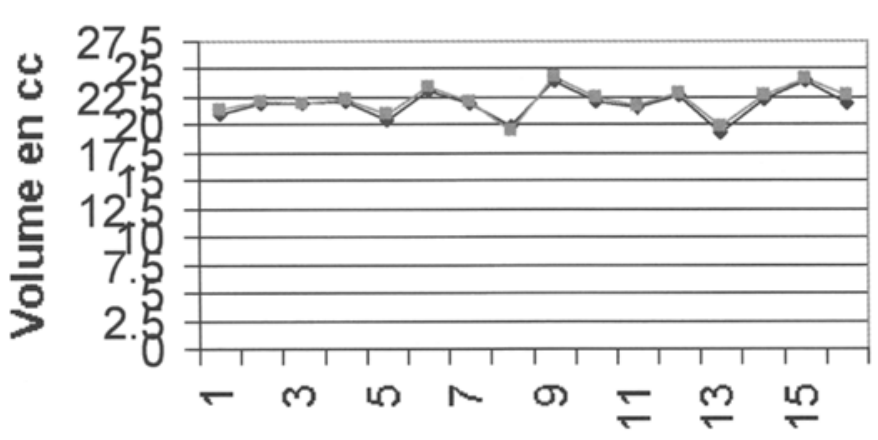

Pts

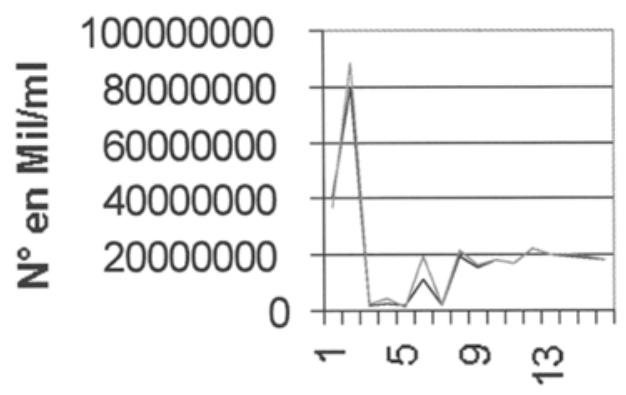

Pts

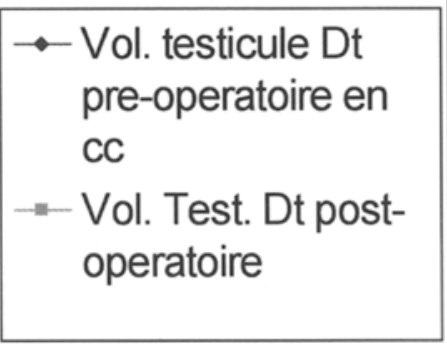

Figure 3A : Distribution du volume testiculaire droit avant et après chi. rurgie.

\begin{tabular}{|l|}
$\rightarrow-$ Vol. testicule Gh \\
preoperatoire en \\
cc \\
-- Vol. Test. Gh \\
post-operatoire
\end{tabular}

Figure $3 B$ : Distribution du volume testiculaire gauche avant et après chirurgie.
Figure 4 : Distribution de la concentration de spermatozoìdes avant et après chirurgie. 


\section{REFERENCES}

1. BELGRANO E., PUPPO P.. QUATTRINI S.. TROMBETTA C., PITTALUGA P. : Microsurgical spermaticoepigastric anastomosis for treatment of varicocele. Microsurgery, 1984. $5: 44-49$.

2. CAYAN S., KADIOGLU A., ORHAN I., KANDIRALI E.. TEFEKLI A., TELLAI_OGLU S. : The effect of microsurgical varicocelectomy on serum lollicle stimulating hormone. lestosterone and free testosterone levels in infertile men with varicocele. Br. J. Urol. Int.. 1999, 84 : 1046-1049.

3. CAYAN S., KADIOGLU T.C., TEFEKLI A., KADIOGLU A., TELLALOGLU S. : Comparison of results and complications of high ligation surgery and microsurgical high inguinal varicocelectomy in the treatment of varicocele. Urology, 2000, $55: 750$ 754.

4. DEBRE B.. POIZART R., LELLEU C.H. : Varicocèle. Encycl. Med. Chir. (Paris) : Rein, organes génito-urinaires. 18648 C/101983.

5. DRAGHI F., FERROZZI G.. CALLIADA F., SOLCIA M.. MELONI G.. CAMPANI R. : Assessment of varicocelc recurrence with ultrasonography. color and power doppler. Radiol. Med.. $1997,93: 572-575$.

6. GOI_DSTEIN M. : Terapia chirurgica dell'infertilità maschile $c$ di altre patologic dello scroto. In "Urologia di Campbell". Roma. Verduci, 1999 (trad. ed. inglese 1998) : 1379-1386.

7. GOLDSTEIN M., GILBERT B., DICKER A., DWOSH J., GNECCO C. : Microsurgical inguinal varicocelectomy with delivery of the testis : an artery and lymphatic sparing technique. J. Urol.. 1992; 148 : 1808-1811.

8. HIRSH A.V., CAMERON K.M., TYLER J.P., SIMPSON J., PRYOR J.P. : The doppler assessment of varicocele and internal spermatic vein reflux in infertile men. Br. J. Urol.. 1986, 135 : 483-484.

9. ISHIGAMI K.. YOSHIDA Y.. HIROOKA M., MOHRI K. : A new operation for varicocele: use of microvascular anastomosis. Surgery. 1970, $67: 620-623$.

10. MIEUSSET R. : Températures et varicocèles. Andrologie, 2002. $12: 91-99$

11. OZBEK E., TURKOZ Y., GOKDENIZ. R.. DAVARCI M.. OZUGURLU F. : Increased nitric oxide production in the spermatic vein of patients with varicocelc. Eur. Urol., 2000, 37 : 172-175.

12. SARTESCHI M., PAOLI R.. BIANCHINI M.. MESCHINI FABRIS G.F. : Lo studio del varicocele con ecocolordoppler. Giorn. It. Ultrason.. 1993. $4: 43-49$

13. SAUTTER T., SULSER T., SUTER H., GRETENER H., HAURI D. : Treatment of varicocele : a prospective randomised comparison of laparoscopy versus antegrade sclerotherapy. Eur. Urol., 2002, $41:$ 398-400.

14. SEYFERTH W., JECHT E., ZEITLER E. : Percutaneous sclerotherapy of varicocele. Radiology, 1981, 139:335-334.

15. STUDENT V.. ZATURA F., SCHEINAR J., VRTAL R.. VRANA J. : Tcsticlc hemodynamics in patients after laparoscopic varicocelectomy evaluated using color doppler sonography. Eur. Urol., 1998, $33: 91-93$.

16. SUN N., CHEUNG T.T., KHONG P.L., CHAN K.L., TAM P.K. : Varicocelc: laparoscopic clipping and color doppler follow-up. J. Pediatr. Surg., 2001, 36: 1704-1707.

17. TASCI A., RESIM S., CASKURLU T., DINCEL C., BAYRAKTAR Z.. GURBUZ G. : Color doppler ultrasonography and spectral analysis of venous flow in diagnosis of varicocele. Eur. Urol. 2001. 39 : 316-321.
18. TEFEKLI A., CAYAN S., ULUOCAK N., POYANLI A., ALP T., KADIOGLU A. : Is selective internal spermatic venograpy necessary in detecting recurrent varicocele after surgical repair? Eur. Urol., 2001, $40: 404-408$.

19. THOMAS A.J. Jr., GEISINGER M.A. : Current management of varicoceles. Urol. Clin. North Am., 1990, $17: 893-907$.

20. WAGNER L. : Varicucèle: physiopathologie et approches thérapeutiques. Andrologie. 2002, $12: 100-104$.

\section{ABSTRACT}

Haemodynamic results and complications after dissection of the spermatic vein in the internal inguinal ring with a $4.5 \mathrm{x}$ operating microscope.

Giandomenico PASSAVANTI, Valerio PIZZUTI,
Fabio Massimo COSTANTINI, Riccardo PAOLINI

Inversion of venous flow is well visualized by colour duplex ultrasound and is the basic haemodynamic feature of varicocele. We tried to assess the value of dissection of branches of the spermatic vein in the internal inguinal ring using an operating microscope with a magnification of $4.5 \mathrm{x}$.

16 patients were operated according to the ultrasound classification proposed by Hirsh et al. [8]. Varicocele was grade III in 2 cases, grade IV in 11 cases and grade $V$ in 3 cases and was situated on the left in 13 patients and on the right in 3 patients.

The diagnosis was based on clinical examination and colour duplex ultrasound. The operating time was 25-35 min. The incision was 1.5 to $2 \mathrm{~cm}$ long. No short-term complications or long-term hydrocele were observed. No reno-spermatic flow was observed on colour duplex ultrasound after six months. The mean testicular volume was $22 \mathrm{ml}$ before surgery and did not change after the operation.

This surgical technique is less invasive than the conventional retroperitoneal or laparoscopic procedures, and the incision is smaller than with the percutaneous technique. It is less radical than conventional microsurgery, but the operating time is shorter, and the surgical wound is smaller, sparing the artery and lymphatic vessels.

In conclusion, this operation can be considered to be a good option in the surgical treatment of varicocele in view of its low complication rate, the absence of hydrocele, a minimally invasive procedure and the good haemodynamic results.

Key-Words: varicocele, varicocelectomy, power-Doppler, microsurgery 